\title{
Analysis of the Treadmill Utilization for the Development of a Virtual Reality Walking Interface
}

\author{
GawnYong Park ${ }^{1}$, Sang Hwa Lee ${ }^{2}$ and JungYoon Kim ${ }^{3 *}$ \\ ${ }^{1}$ Graduate School of Game, Gachon University, 1342 Seongnam Daero, Sujeong- \\ Gu, Seongnam-Si, Gyeonggi-Do, South Korea, 461-701 \\ ${ }^{2}$ Scool of Manhwa Contents at Chungkang College of Cultural of Industries, \\ Majang-myeon Gyeonggi-Do, South Korea \\ ${ }^{3}$ Graduate School of Game, Gachon University, 1342 Seongnam Daero, Sujeong- \\ Gu, Seongnam-Si, Gyeonggi-Do, South Korea, 461-701 \\ 1gawn1@naver.com, ${ }^{2}$ gomawooi@naver.com, ${ }^{3}$ kjyoon79@gmail.com
}

\begin{abstract}
Virtual reality (VR) has been used in various fields and recent applications of virtual reality technology include education, medicine, science, and tourism. In particular, virtual reality technology has been used most for experience based virtual reality contents and game contents. Sometimes called Virtual Environments (VE), it has drawn much attention in the last few years due to extensive media coverage however few people really know what VR is, what its basic principles and its open problems are. One of the important issues in developing such virtual reality experience contents is human computer interface which can be designed and operated in such a way to meet a purpose such as walking over long distances. However, virtual reality technology with the use of tracking devices and cabling has has until recently restricted these abilities of users. Walking interface provides an interactive human-computer interaction that conveys proper walking sense to users by receiving and processing walking speed of users. This paper studies walking interface hardware out of controls under virtual environment that provides users with the ability to experience real-life walking actions. Specifically, this study aims to examine the existing walking interface and to introduce treadmill based simple biped ambulation interface using pressure mode sensor. Walking interface enables researchers to seek space in virtual environment by using natural walking which can substitute the above interface. And the use of interface such as joystick is used to seek space in virtual environment. The stepping actions are performed on top of a treadmill with embedded grid of switch sensors that detect footfalls pressure. The overall state of the treadmill based interface is scanned at a rate of $100 \mathrm{~Hz}$ which can deliver real-time visual feedback reaction to user actions and steps.
\end{abstract}

Keywords: Virtual Reality, Human-Computer Interaction, Walking Interface

\section{Introduction}

The development of virtual reality (VR) in 2017, head mounted display (HMD) technology to maximize immersion has advanced much. Numerous number of hardware manufacturers have invested lots of money in developing virtual reality device. However, virtual reality contents are insufficient to enhance immersion and to lessen cognitive dissonance phenomenon by using HMD hardware only because an experience is different from reality. Accordingly, numerous business enterprises have researched and developed

Received (December 6, 2017), Review Result (February 5, 2018), Accepted (February 12, 2018)

* Corresponding Author 
additional hardware other than head mounted display [1]. Research to develop hardwarebased technologies that can reduce user's cognitive dissonance and enhance immersion level and recognize and process user's intention to move in virtual reality is under way [2].

Virtual reality provides the capability to build virtual space with high level of immersion in such a manner that it is possible to seek virtual space by using human walking. Walking is considered to be an important life activity which is accepted as a natural behavior that human performs to get from place to place. It is a fundamental requirement for action and navigation in one's daily life. Keeping such an active and dynamic ability to move through large-scale virtual environments (VEs) is of great interest for many applications demanding locomotion, such as building evaluation, urban planning, terrain exploration, etc. However, user movements in virtual environments are usually restricted by a limited range of tracking sensors, and constrained by the small workspace of the real world. Thus, some kind of virtual ambulation interface is needed to enable movements over large distances while remaining within a relatively small physical space. Also, one of the major shortcoming of virtual reality systems with large screen nowadays is the presence of an open area where no visual feedback cue can be available. This area is usually located at the opposite side of the center of the display and behind the user. The challenging part in integrating ambulatory interfaces for VE with large screens has been always how to let users perform comfortable and effective life-like navigation without being physically displaced outside the limited area of the interface.

To address these issues, this study proposes and designs and implements motion based walking interface to build virtual space with high level of immersion in such a manner that it is possible to seek virtual space by using human walking. In addition, the author uses interface such as joy stick to seek space to build virtual space. Findings of this study are expected to be used when specific task should be performed while understanding size or complexity of a facility which walking speed is important [3].

An obvious approach to implement real walking is to transfer user's tracked head movements to changes of the virtual camera in the VE by means of a one-to-one mapping, wherein a one meter movement in the tracked space is mapped to a one meter movement of the virtual camera in the corresponding direction. This technique has the drawback that the users' movements are restricted by a limited range of the tracking sensors and a rather small workspace in the real world. Therefore, concepts for virtual locomotion are needed that enable walking over large distances in the virtual world while remaining within a relatively small space in the real world. Various prototypes of interface devices have been developed to prevent a displacement in the real world [4][5]. Although these hardware systems represent enormous technological achievements, they are still very expensive and will not be generally accessible in the foreseeable future.

The remainder of this paper is structured as follows. In chapter 2, related literature in the development of virtual reality walking interface is discussed. In chapter 3 , walking interface design and implementation are elaborated in detail. In chapter 4, conclusion and future research are presented.

\section{Related Research}

Virtual reality involves real-time interactive graphics with three-dimensional models, combined with a display technology that gives the user the immersion in the model world and direct manipulation [6]. Also, the illusion of participation in a synthetic environment rather than external observation of such an environment. VR relies on a threedimensional, stereoscopic head-tracker displays, hand/body tracking and binaural sound. VR is an immersive, multi-sensory experience [7].

Creating a high fidelity virtual reality experience is difficult mainly because of technical limitations it presented. Basic locomotion requirements like support for omni- 
directional and unlimited walking often interfere with these. As such, many research groups focuses locomotion in a large virtual world by real walking through a limited physical setup. Walking interfaces with different locomotion interfaces using similar principle of treadmill systems were proposed by [8][9]. This principle incorporates the goal of cancelling users' displacement and keeping them located at the same place while being able to walk into any direction. The design of these systems primarily include head mounted display for visual feedback. They also carry a heavy weight of more than 100 kilograms, and use a set of attachments to the body of the users related to tracking sensors and safety mechanisms. However, the use of head mounted display caused some safety concern so a large display system is suggested [10] so that it could be more suitable for the interface.

In addition, an approach to walking interface is to scale translational movements in order to cover a virtual distance that it is larger than the distance walked in the physical space [11]. Similarly, a simpler approach that used the stepping actions to engage user into a walking experience were proposed by [12][13] [14]. These systems were implemented with HMD and cabled sensors to read user's stepping behavior, but the use of HMD and cabled sensor to the user body make them inconvenient for integration in virtual environment with large display where cables may disturb user's movements. Moreover, walking interface without the use of a HMD but with an interesting redirection technique that keeps user oriented toward the screen was used by [15]. This allows a user to walk around virtual environment without losing sight of projected images.

Linear walking systems have been developed to reproduce active walking experience within virtual environment with the use of bi-pedal interface through linear treadmill [16]. Yet these interfaces do not provide users with the ability to use their active and natural body walking action. Several virtual reality systems such as CyberSphere and Cabine VR [17][18] deliver surrounding visual feedback cue. However, their bulky structure, space allocation, and high price make them not so popular for virtual reality applications.

\section{Methodology}

Due to its wide ranging potential, VR technology is not defined or limited by any technological approaches or hardware setups. The creation of a virtual reality experience can be accomplished using combinations of a wide variety of interaction devices and sensory display systems and in the design of content presented in a computer-generated graphic world. Particularly, immersive virtual reality combines computers, head-mounted displays, body-tracking sensors, specialized interface devices, and real-time graphics to immerse a participant in a computer-generated simulated world that changes in a natural way with head and body motion. As such, one of the key aims of these systems is to replace the outside world perceptually with that of a simulated environment to create a specific user experience.

For walking interfaces under virtual environment, ground moving mode and pedal based moving mode that moves by tracking moving foot are common [19]. For ground moving mode, treadmill composed of belt and a pair of rollers is used mostly. The treadmill is equipped with turntable so that treadmill can rotate to change a direction. Thus, in this study, the proponent introduces treadmill based simple biped ambulation interface integrated with pressure mode sensors.

\subsection{Design of Walking Interface}

The proposed virtual reality walking interface system as illustrated in Figure 1, is composed of these major components: a walking interface platform using treadmill, head mounted display, a sensing system, and a large display. A treadmill based walking interface with sensors is used as walking interface which the user will interact with virtual environment being projected on the large screen. In order to engage into a virtual walking 
experience, users need to perform a stepping actions without propelling their body. Stepping actions are detected in real-time through a series of sensors and treated as a gesture of moving forward in the virtual environment.

The physical environment for the virtual reality walking interface using a treadmill is a $12 \mathrm{~m} \times 8 \mathrm{~m}$ laboratory room. The user wears a head mounted display with the following specifications (3DVisor Z800 with a 40\# diagonal field of view) on which the virtual environment is rendered.

Pressure mode sensors are scanned at a rate of $100 \mathrm{~Hz}$ allowing therefore a real-time tracking of user's walking behavior on the treadmill. As soon as the system recognizes a moving pattern, projected images representing virtual environment will be updated accordingly. The walking interface that can be integrated into a wide variety of virtual reality systems are equipped with large display system. The interface employs a walking platform through treadmill to impart users with the ability to freely engage in a real walking experience into any direction without losing sight of projected images even with the use of limited screen size.

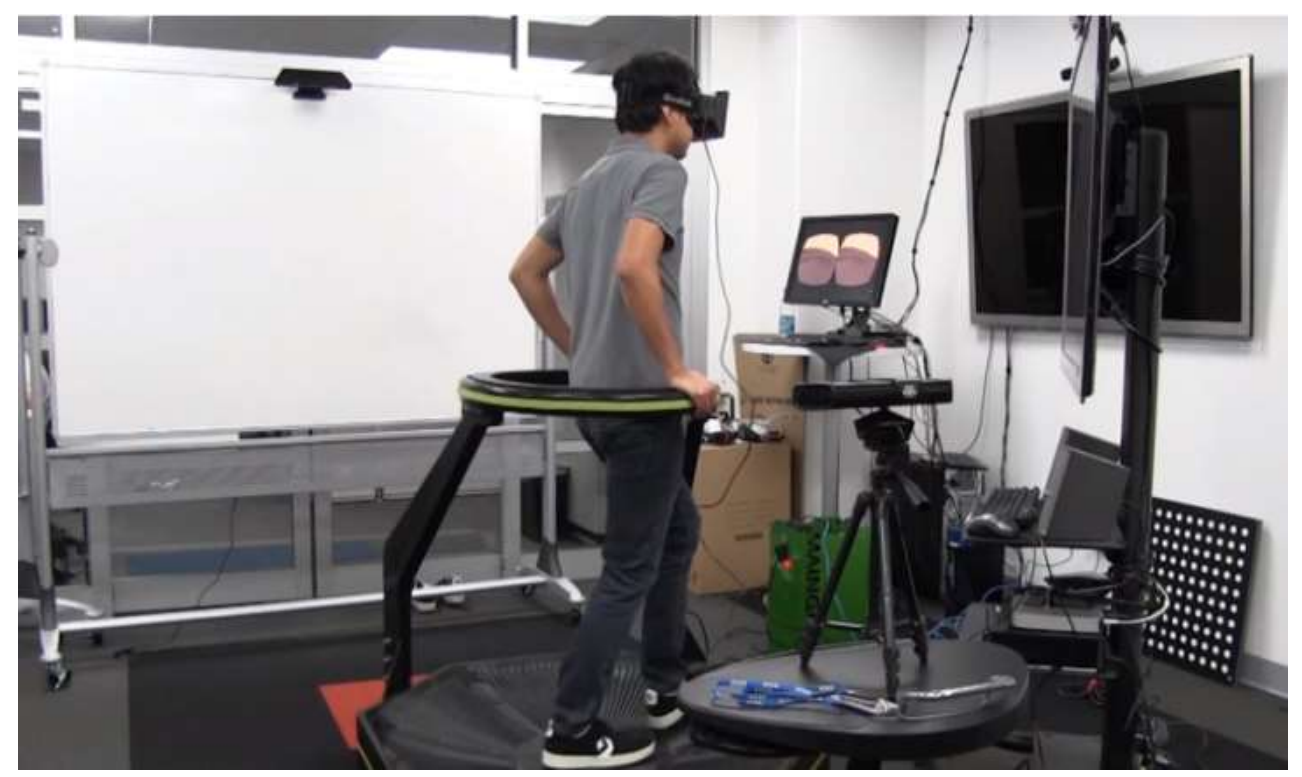

Figure 1. Construction of Treadmill based Virtual Environment

Head mounted displays are headsets incorporating two small cathode ray tube (CRT) or liquid-crystal display (LCD) monitors placed in front of the user's eyes. A tracker measures the images that are presented to the user based on his/her current position and orientation. Due to mounting of HMD to the user's head, head mounted displays are required to fulfill strict ergonomic requirements in order to reduce user fatigue, discomfort and injury. These requirements include the following: it should be relatively light, comfortable and easy to put on and off. As any visual display it should also have possibly the best quality. These demands force hardware engineers to make hard tradeoffs between cost and quality. As a result, the prices and quality of head mounted displays vary dramatically, from a low-cost, low-quality device to high-tech military head mounted displays [20]. Relatively, the use of opaque HMDs totally replaces the user's view with images of the virtual world and can be used in applications, that create their own world such as architectural walkthroughs, scientific visualization, and games. Most of the HMDs currently available on the market support these various applications.

\subsection{Attributes of Walking Interface}


The proposed walking interface is designed to support the following attributes. Firstly, it is designed to be body centered. This allows the ambulatory actions controlling the navigation to be initiated and sustained by the lower part of the body as in real life situation. This approach will preserve user's natural reflexes and navigational control skills. Moreover, user's hands are free for manual interaction in this type of setup. Secondly, the design of the walking interface is simple and easy to use. The walking interface is easy to set up, easy to learn, and easy to use which provide the capabilities to decrease the mental and physical workload brought by the interface and to increase the sense of presence in the virtual environment. Thirdly, the design is robust and compact wherein the hardware system fits most of virtual reality system with large display. Finally, user walking techniques are omni-directional where they can guide their steps in any direction.

\subsection{Requirements of Walking Interface under Virtual Environment}

Walking interface requires technologies of walking through linkage of software and hardware, motion recognition and sensor so that a user can experience with head mounted display. The required technologies are presented in Table 1. These technologies provide the basis for the implementation of the walking interface.

Table 1. Requirements for Walking Interface under Virtual Environment

\begin{tabular}{|l|l|}
\hline Category & Required technologies \\
\hline $\begin{array}{l}\text { Video } \\
\text { technology }\end{array}$ & $\begin{array}{l}\text { HMD Oculus library implementation technology } \\
\text { Real time rendering system technology } \\
\text { Underground life modeling real motion implementation } \\
\text { technology }\end{array}$ \\
\hline Hardware & $\begin{array}{l}\text { Window based operation hardware production technology } \\
\text { Walking hardware design and production technology } \\
\text { Motion recognition and other USB hardware soft library } \\
\text { connection technology }\end{array}$ \\
\hline HMD hardware & $\begin{array}{l}\text { HMD linkage technology via engine which video technology and } \\
\text { hardware technology are integrated }\end{array}$ \\
\hline
\end{tabular}

The video technology is aided by the recent innovations by Sony and Oculus VR which includes virtual reality head mounted that are available at consumer-level prices. The realtime rendering system technology focuses on producing and analyzing images used in reference to interactive 3D computer graphics, typically using a graphics processing unit (GPU). The underground life modeling real motion technology will provide virtual environment relevant to doing underwater activity.

Software and hardware technologies required to design walking interface under virtual environment are described and walking interface is designed and implemented based on technologies specified in the table. This chapter describes the existing virtual environment contents and differentiation when producing contents by implementing walking interface.

\subsection{Implementation of Walking Interface}

As shown in Figure 2, column equipped with three axis noise free cylinder and center fixed type base are set automatically and walking machine to be installed at the bottom has pressure sensor to prevent inconvenience that a user operates manually for walking interface design under virtual environment. Sensor was designed so that users can experience walking interface by recognizing pressure regardless of their weight. The size of round bar was expanded to secure safety while walking machine turns 360 degrees. 
Walking machine that is equipped with pressure sensor at the bottom is designed so that shock from load of 160 kilogram can be absorbed.
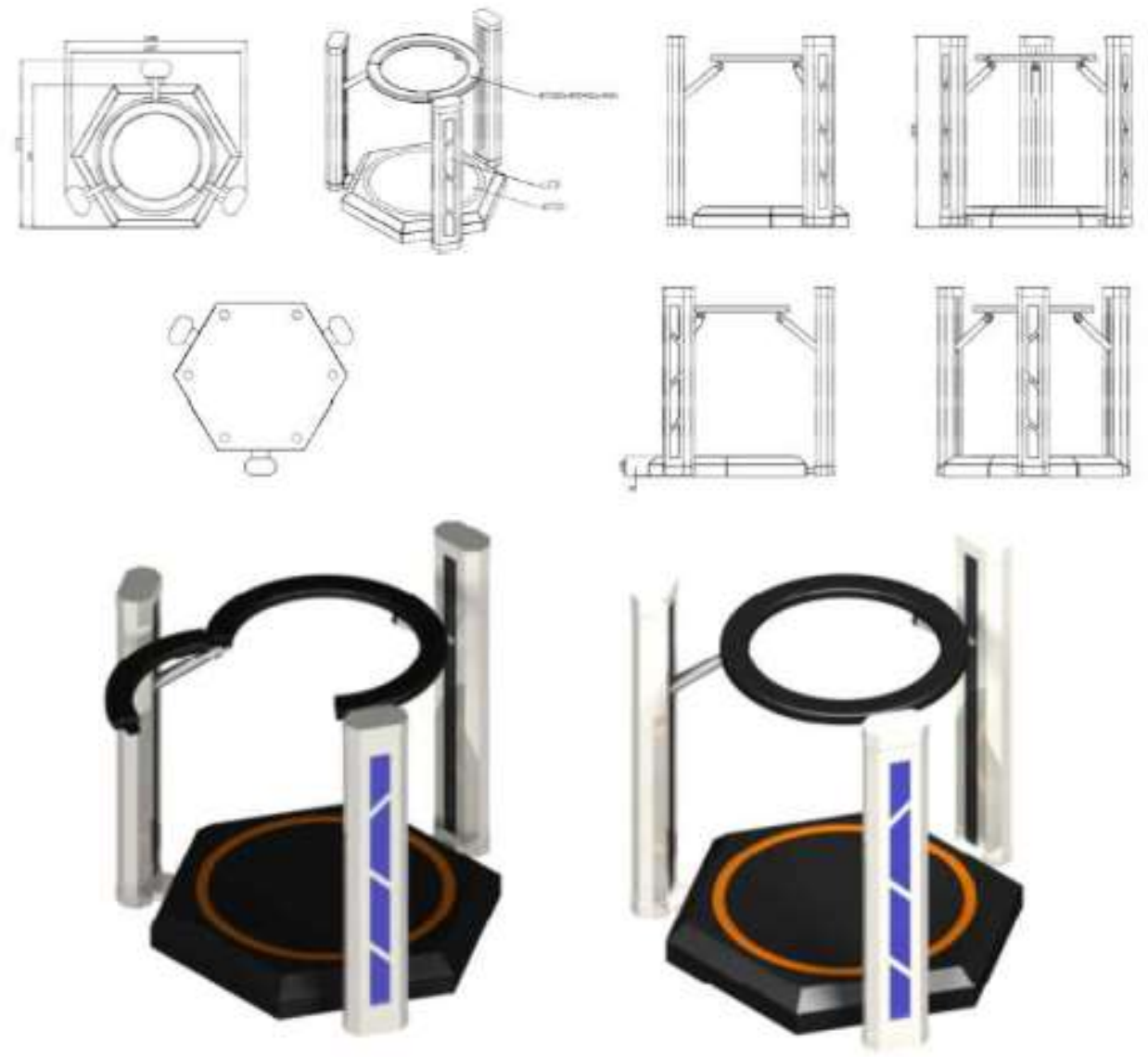

Figure 2. Virtual Environment Construction Walking Interface Design

\subsection{Visual Display Transformation}

The visual display transformation for virtual reality is much more complex than in standard computer graphics. To provide a proper view of the scene, the components are to be taken into consideration such as the hierarchical scene database containing a number of objects and the user controlling the virtual camera by moving his/her head, flying through the world, or manipulating it. The determination of viewing parameters involves the calculation of a series of transformations between coordinate systems that depend on hardware setup and state of input devices.

To render the visual images, the camera in the virtual world is needed to be determined. Thus, following transformations must be calculated by the eye in the sensor which defines the position of the eye from the virtual camera in the tracker's sensor coordinate systems. These transformations for both the left and right eye are fixed for a given head mounted geometry although different HMDs can have tracker's sensors mounted differently. To resolve the final viewing transformation from the object coordinate system into the screen coordinate system, the viewing perspective projection and object in the world transformations like in standard computer graphics is taken into account (see Figure 3). 


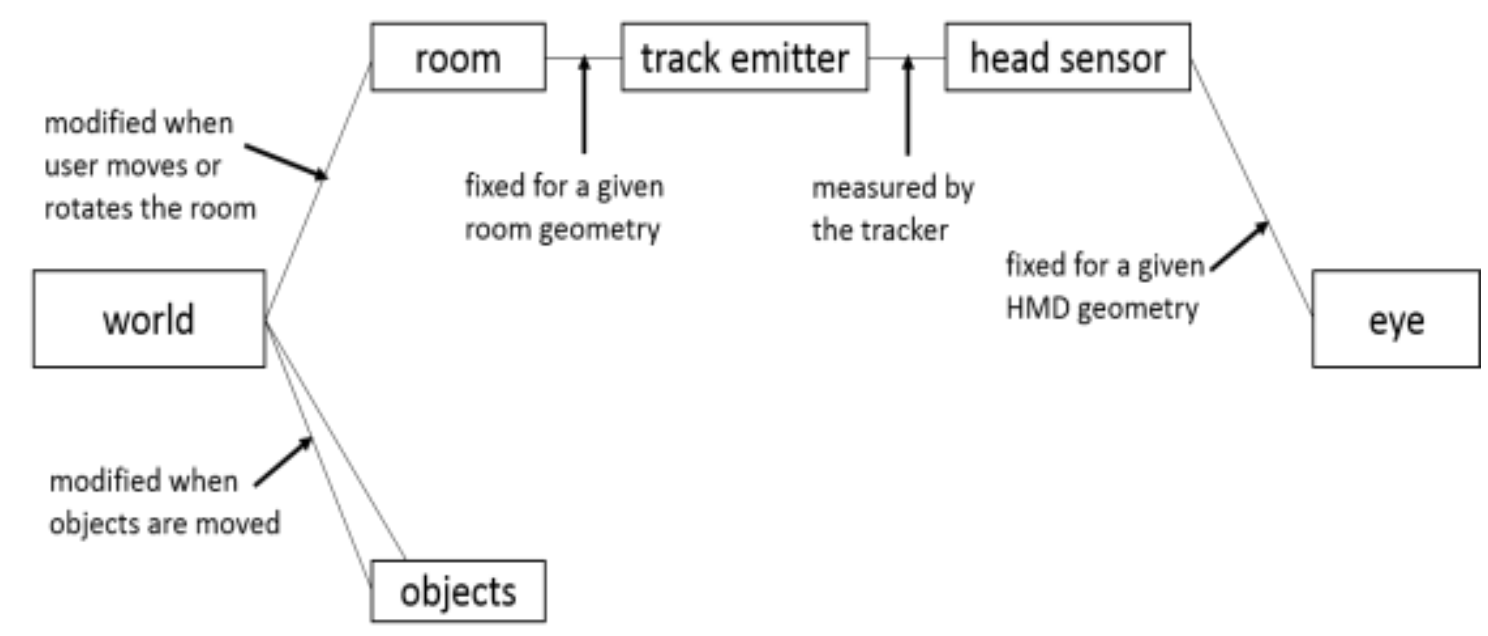
Figure 3. Coordinate System Transformations for Virtual Reality (Adapted
from [21])

In addition, proper pairs of visual images called as stereo pairs should be generated for stereo perception in computer graphics. For a generation of stereo images this method uses parallel viewing rays for each of the eyes and the perspective projection (See Figure 4).

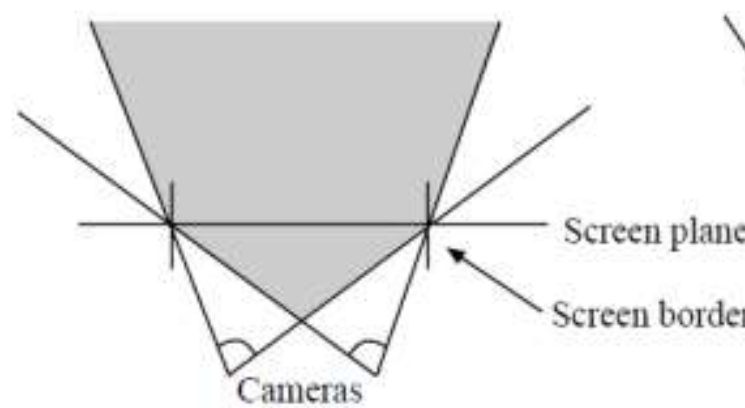

(a)

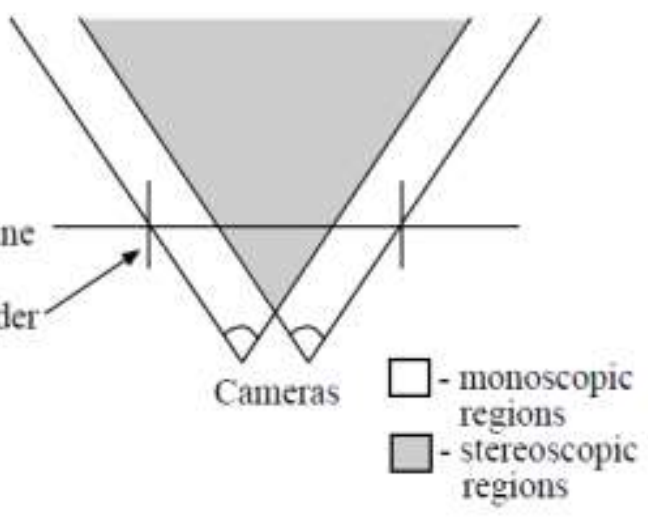

(b)

Figure 4. Two Centers of Projection Transformations: (a) Off-Axis Projection, (b) on-Axis Projection ([22])

\subsection{Level of Immersion}

In a virtual environment system, a computer generates sensory impressions that are delivered to the human senses. The type and the quality of these impressions determine the level of immersion and the feeling of presence in virtual reality. Ideally the highresolution, high-quality and consistent over all the displays, information should be presented to all of the user's senses [23]. Moreover, the environment itself should react realistically to the user's actions. The practice, however, is very different from this ideal case. Many applications stimulate only one or a few of the senses, very often with lowquality and unsynchronized information. The level of immersion this study offers to users is an immersive system. It is considered as the ultimate version of virtual reality systems due to capabilities of letting the user totally immerse in computer generated world with the help of head mounted displays that supports a stereoscopic view of the scene 
accordingly to the user's position and orientation. The system may be enhanced by audio, haptic and sensory interfaces.

Immersive virtual reality has been most commonly employed in applications where a controlled stimulus environment is desirable for constraining a user's perceptual experience within a specific synthetic world. By contrast, non-immersive virtual reality is commonly experienced using modern computer and console game systems. This format presents a three-dimensional graphic environment on a flatscreen monitor or television wherein there is no realworld occlusion, within which the user can navigate and interact. Despite delivered on a less immersive display, such graphic worlds are still essentially a virtual reality environment.

Virtual environments presented on these widely available commodity display systems have the capacity to provide the user with significant options for interaction with dynamic digital content using traditional computer and game interface devices such as keyboard, mouse, game pads, joysticks, etc. The use of such ubiquitous display and interface devices has promoted widespread access to this form of non-immersive interactive media, mainly in the domain of entertainment. Moreover, researchers have investigated the value and usability of commercially available interaction devices and methods that can be used with flatscreen-delivered VEs that can allow users to interact with digital content using more naturalistic body actions beyond what is possible with traditional game interfaces such as Konami Dance Dance Revolution, Sony Eyetoy, Nintendo Wii, and Microsoft Kinect. Regardless of the hardware format, the capacity of VR technology to create controllable, multisensory, interactive three dimensional stimulus environments within which human performance can be motivated, recorded, and measured, offers clinical assessment and intervention options that are not possible using traditional methods. Much like an aircraft simulator can serve to test and train piloting ability under a variety of controlled stimulus conditions, VR can be used to create relevant simulated environments that allow for the assessment and treatment of cognitive, emotional, and motor functioning. Such VR simulations can afford many assets to both clinical and research methods that are not available with traditional approaches. A full detailing of these assets such as ecological validity, controlled stimulus environment, naturalistic performance recording, safety, tailored feedback are published elsewhere.

\subsection{Walking Actions}

The different actions that the system can detect and treats as voluntary moving gestures are: stepping, turning, and jumping. All these actions are tracked by analyzing the different contact interaction between the feet and the walking-pad platform. In other term, matching the patterns presented by active sensors and user actions. For this aim, finding the center of gravity of the activated sensors is a consistent and reliable indicator that can be used to predict user's stepping behavior on top of the treadmill. Furthermore, the center of gravity gives also an idea on the placement of footfalls on the platform.

The application of walking detection is based on a state-of-the-art low-latency walk phase detection algorithm [24] that successfully used for a walking-in-place technique [25]. The recognition algorithm identifies the current step section of the leg movement using a series of optional conditions that need to be matched. These conditions include a minimum and maximum acceleration in each direction wherein a maximum amount of time the previous step section is valid. In this scenario, a masking time in which no next step section may be detected and a minimum and maximum difference between the two vital accelerations corresponding to vertical and depth. A step is only completed when each of the step sections occur consecutively.

The frequency of step is transformed to walking speed using the heuristics of the walk technique. With small adjustments, the resulting predictions to implement a very low latency starting, stopping and walking speed detection is utilized. In this working prototype, we achieved reasonable immersion with one large planar display. However, 
this only allows relative orientation gestures. Thus, to support absolute orientation, a more immersive cave setup or heads-up display is necessary.

\subsection{Analysis of Walking Interface under Virtual Environment}

Walking interface in this study was developed to optimize HMD Oculus experience hardware development. Computer graphic, three dimensional video and virtual reality contents production technologies are required to develop a technology. An advantage of walking interface developed in this study and existing walking interfaces is that walking interface developed in this study is safer than existing walking interfaces when a user experiences contents and walking machine turns 360 degree and a user can start experience more easily and quickly after entering tread mill without wearing other hardware. Figure 5 is a screen showing virtual environment construction walking interface experience. It shows the view of a player in the virtual environment doing underwater activity. This was also used for the psychophysical experiments and the evaluation of transitional environments.

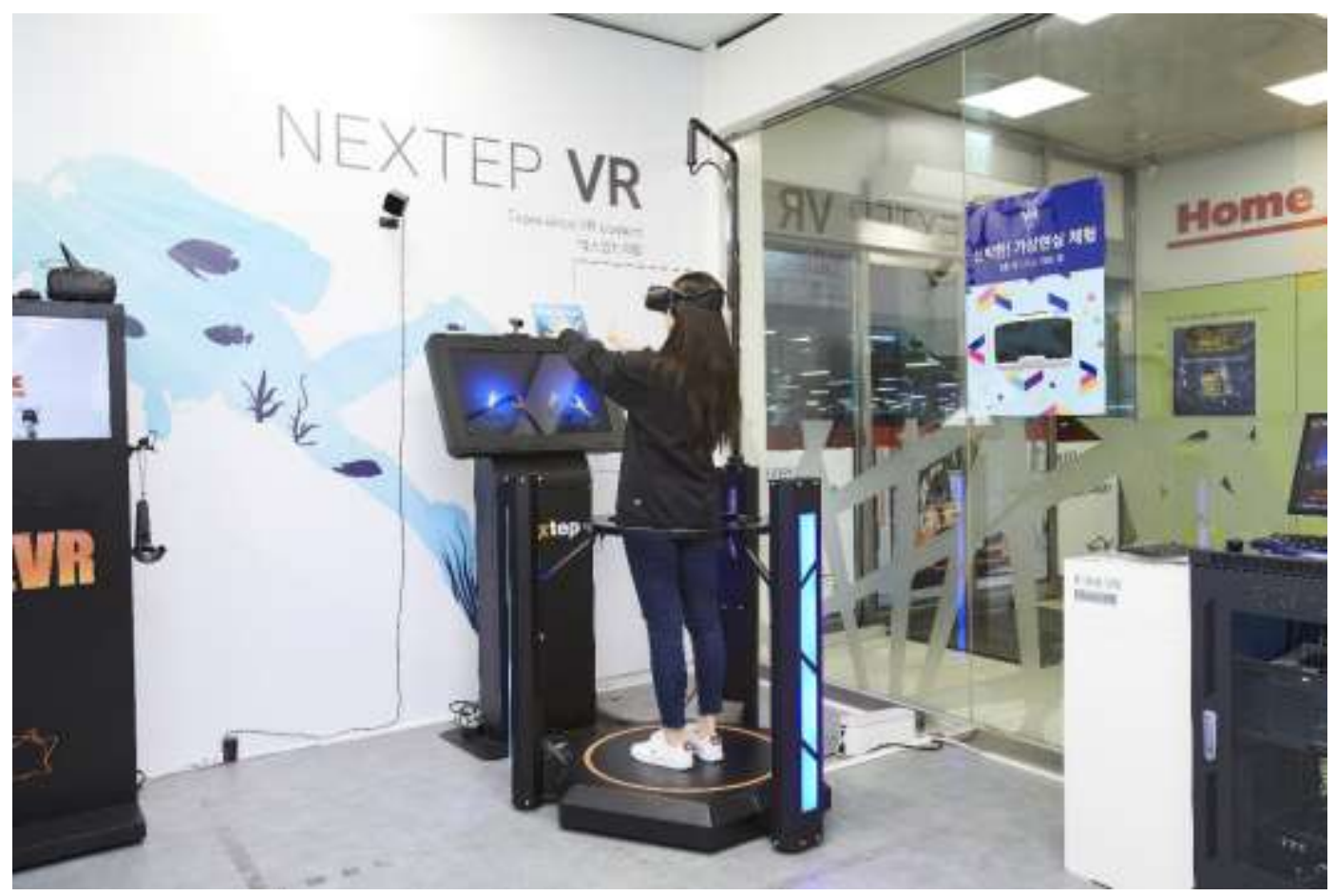

Figure 5. Screen Showing Virtual Environment Construction Walking Interface Experience

The objective of this experiment is to support omni-directional and unlimited walking with a geospatial context. In order to ensure that players do not observe that they get manipulated, a psychophysical experiment in which subjects had to discriminate the walk direction wherein subjects had to judge whether the walked curve is bent to the left or to the right is performed. The visual stimulus used in the experiment the walking interface was generated.

In order to verify if a virtual environment increases the participant's sense of presence in this experiment, the proponent have conducted the experiment with according to the condition that subjects started in the virtual model when they turned on the HMD, so they started the underwater based virtual game directly from the real world. After having been equipped with the HMD, participants saw a realistic model of the laboratory space used as virtual environment. In the scenario, participants could talk with the aid of the experiment 
assistant while they participants are walking through treadmill in the virtual environment. Thus allows the communication to indicate that they are still not in the virtual world. After approximately 5 minutes the participants are told to had to press a particular button on the remote device in order to open a portal to the virtual world as shown in Figure.

\subsection{Physical Simulation}

Virtual reality may be considered as a clone of physical reality or it provides representation of something closer to cyberspace that has defines its own rules. In these cases, a physical simulation of the virtual environment has to be done to realize its efficiency and application of walking interface. In the case of newly defined cyberspace, the task is relatively easy in which one can invent new laws or use physics for the interoperability of the walking interface using treadmill. The real challenge is to simulate the rules of physics because they are very complex phenomena as it involves dynamics of the walking interface components, the operation of electromagnetic forces, etc., that works within the simulation of the interface. For the human-computer interaction purposes, a subset of the walking interface components has to be considered. In these, Newton's laws are the basis when simulating user movements, and force interaction among the user and the components of the walking interface [26].

The simulation and animation of components may be a very complex and timeconsuming task. The simulation process that manages the behavior of the whole environment should be run in the background in order to support the full performance and efficiency. The updates between the application parts are realized by means of user operations that are considered asynchronous.

Thus, the construction and maintenance of physically based, multi-user and distributed virtual environment is not an easy task. Beside usual expectations such as high efficiency support for lag minimization, it demands hardware independence, flexibility and highlevel paradigms for easy programming, maintaining and consistent walking interface.

\subsection{Evaluation}

To get information from the approach being used, the proponent evaluated the virtual reality walking interface based on treadmill in the participants' test. 20 test persons aged between 20 and 32 (average 25.83) took part in the experiment of testing the applicability and efficiency of the virtual reality walking interface. 16 of the participants were male, 4 of them were female. Most subjects are students or members of the departments (computer science, mathematics, psychology, geoinformatics, and physics). All have normal or corrected to normal vision; 8 wear glasses or contact lenses. In addition, 8 have no game experience, 4 have some, and 6 have much game experiences. The total time per subject including instructions, training, and debriefing took 20 minutes and was performed within two days in all. Then the data gathered from the survey questionnaires were organized, collated and stored into the computer for analysis.

Each participant used each technique to play an exergame adaptation of simulating portal in an underwater diving activity with interaction to fishes. The experiment consisted of a mission course specifically designed for the experiment with feeding the fishes. While at first the participants found the setup not really comfortable using it, 15 of them would use it again and 5 rated it to be the most natural and intuitive user interface. Also, most of the users found that using the walking interface with biped treadmill is easier to use. Some of the test persons criticized that contrary to walking-in-place, the treadmill base setup did not allow walking backwards.

To summarize the results, we suggest using treadmill based walking interface for immersive virtually reality environments since they have great potential to enhance experience and presence, while portals to the virtual world can further improve the virtual 
reality experience. The result of experiment indicated that a positive reaction from the participants was observed in the virtual reality game contents experience.

\section{Conclusion}

This study developed walking interface system that can perform walking navigation under virtual reality environment by recognizing motion of a walker using pressure sensor. A user can start experience contents easily and quickly entering tread mill without wearing other hardware. Walking interface developed in this study is equipped with automatic speed control system that can control walking speed, which makes it possible for a user to feel immersion. Walking interface was designed so that malfunction can be minimized by controlling moving speed in tread mill and location and direction are recognized from the view of a user.

Walking system for virtual reality navigation with high immersion was constructed by providing walking experience or training environment in a limited space and simulating physical contact process with ground. It is advisable that future research should cover development of algorithm such as motion tracking and recognition for improvement of walking interface, hardware response speed and safety which is concerned with inertia.

Every virtual reality application must be effective by means of performance and interaction. This requirement can be only fulfilled when all parts of the virtual reality system are properly integrated one with the other. Nowadays, even the best hardware cannot support this by itself as such it needs software assistance for precise control, resources management, and synchronization.

For future work, the proponent would like to investigate the ability to integrate haptic interface with the current system so as to impart users with the ability to physically interact with virtual environment during their traveling. Specifically, the walking interface is being envisioned to be a wireless interface and include other locomotion features such as sidestepping and backward movement. Another interesting improvement will be to dynamically control the rate of displacement and rotation. For now step size and rotation rate are both fixed in advancement. A possible approach is to use footfall size and increase the resolution of the sensors used.

\section{Acknowledgments}

This research is supported by Ministry of Culture, Sports and Tourism(MCST) and Korea Creative Content Agency(KOCCA) in the Culture Technology(CT) Research \& Development Program 2017 (R2017030062_00000001).

\section{References}

[1] O. Merhi, "Motion sickness, console video games, and head-mounted displays", Human Factors, vol. 49, no. 5, (2007), pp. 920-934

[2] M. Slater, M. Usoh and A. Steed, "Taking steps: the influence of a walking technique on presence in virtual reality", ACM Transactions on Computer-Human Interaction (TOCHI), vol. 2, no. 3, (1995), pp. 201-219

[3] R. A. Ruddle and S. Lessels, "The benefits of using a walking interface to navigate virtual environments", ACM Transactions on Computer-Human Interaction (TOCHI), vol. 16, no. 1, (2009), p. 5 .

[4] L. Bouguila and M. Sato, "Virtual Locomotion System for Large-Scale Virtual Environment", In Proceedings of Virtual Reality, IEEE, (2002), pp. 291-292.

[5] H. Iwata, Y. Hiroaki and H. Tomioka, "Powered Shoes", SIGGRAPH 2006 Emerging Technologies, (2006), p. 28.

[6] H. Fuchs and G. Bishop, "Research Directions in Virtual Environments", NFS Invitational Workshop, Univ. North Carolina, Available also as: ftp://ftp.cs.unc.edu./pub/technical-reports/92-027.ps.Z, (1992).

[7] M. Gigante, Virtual Reality: Definitions, History and Applications. "Virtual Reality Systems", Academic-Press, ISBN 0-12-22-77-48-1, (1993), pp. 3-14. 
[8] H. Iwata and T. Fuji, "Virtual perambulator: a novel interface device for locomotion in virtual environment"; Proc. of IEEE VRAIS'96, (1996), pp. 60-65.

[9] D. C. Brogan, R. A. Metoyer and J. K. Hodgins, "Dynamically simulated characters in virtual environments", SIGGRAPH7 Visual Proc, (1997), p. 216.

[10] J. R. Ensor and G. U. Carraro, "Pelton: A VRML-based bicycling simulator", In SIGGRAPH'97 Visual Proc., (1997), p.198.

[11] V. Interrante, B. Riesand and L. Anderson, "Seven League Boots: A New Metaphor for Augmented Locomotion through Moderately Large Scale Immersive Virtual Environments", In Proceedings of Symposium on 3D User Interfaces, IEEE, (2007), pp. 167-170.

[12] M. Slater, M. Usoh and A. Steed, "Taking steps: the influence of a walking technique on presence in virtual reality”, ACM Trans. On conputer-Human Interaction, vol. 2, no. 3, (1995), pp. 201-219.

[13] M. Usoh, K. Arthur, M.C. Whitton, R. Bastos, A. Steed, M. Slater and F.J. Brooks, "Walking>Walkingin-place>Flying, in virtual environments", SIGGRAPH'99 computer graphics proc., (1999), pp. 359-364

[14] J.N. Templeman, P.S. Denbrook and L. E. Sibert, "Virtual Locomotion: walking in place through Virtual Environments", Presence, vol. 8, no. 6, (1999), pp. 598-617.

[15] S. Razzaque, D. Swapp, M. Slater, M. C. Whitton and A. Steed, "Redirected Walking in Place", Eight Eurographics Workshop on Virtual Environments, (2002).

[16] W. Couvillion, R. Lopez and J. Ling, "The Pressure Mat: A New Device for Travering Virtual Environments Using Natural Motion", Proc. of the Interservice/Industry Training Simulation and Education Conference, (2001), pp.199-211.

[17] http://www.vr-systems.ndtilda.co.uk/sphere1.htm.

[18] T .Ogi, T. Yamada and M. Hirose, "CABINet: Networking of Immersive Projection Environment", HCI: Communication, Cooperation, and Application Design (Proc. HCI'99), pp.1025-1029, Lawrence Erlbaum Associates Press, (1999).

[19] H.-S. Park, "Development of a VR-based treadmill control interface for gait assessment of patients with Parkinson's disease", Rehabilitation Robotics (ICORR), 2011 IEEE International Conference on. IEEE, (2011).

[20] 20.R. Holloway and A. Lastra, "Virtual Environments: A Survey of the Technology”, SIGGRAPH'95 Course, no. 8, (1995), pp. A.1-A.40

[21] M. Mine, "Virtual Environment Interaction Techniques”, SIGGRAPH'95 Course, no. 8 , (1995).

[22] H. Scheirich, "Stereoscopics - Principles and Techniques. Diploma Thesis", ViennaUniversity of Technology, Austria, (1994).

[23] M. Slater, M. Usoh and A. Steed, "Depth of Presence in Virtual Environments", Presence, vol. 3, no. 2, (1994), pp. 130-144.

[24] J.D. Wendt, M.C. Whitton and F.P. Brooks, "GUD WIP: Gait-understanding-driven walking-inplace", In: Virtual Reality Conference 2010. IEEE, (2010).

[25] B. Walther-Franks, D. Wenig, J. Smeddinck and R. Malaka, "Sportal: A first-person videogame turned exergame", In: Proc. Mensch und Computer 2013. Oldenbourg Verlag, (2013).

[26] J. Vince, "Virtual Reality Systems", Addison-Wesley, ISBN 0-201-87687-6, (1995).

[27] J. Fang, D. Clark and J. Simmons, "Collision Detection Methodologies for Rigid Body Assembly in a Virtual Environment", Virtual Reality: Research, Development and Application, vol. 1, no. 1, (1995), pp. 41-48. 